\title{
Simulation of gated GaAs-AlGaAs resonant tunneling diodes for tunable terahertz communication applications
}

\author{
V. P. Georgiev, SMIEEE, A. Sengupta, SMIEEE, P. Maciazek, O. Badami, C. Medina-Bailon, \\ T. Dutta, F. Adamu-Lema, A. Asenov, FIEEE \\ Device Modelling Group \\ James Watt School of Engineering \\ University of Glasgow, Glasgow G12 8QQ, United Kingdom \\ E-mail: vihar.georgiev@glasgow.ac.uk
}

\begin{abstract}
In this work, we report simulations on a GaAs-AlGaAs gated nanowire resonant tunneling diode (RTD) for tunable terahertz communication applications. All calculations are performed with the self-consistent Non-Equilibrium Green's Function (NEGF) quantum transport formalism implemented in our in-house Nano-Electronic Simulation Software (NESS). Our simulations successfully capture the detailed picture of the quantum mechanical effects such as quantum confinement and resonant tunneling of electrons through barriers in such structures. Moreover, we report for the first time the correlation between the gate-bias voltage and the position of the resonant peak $\left(V_{R}\right)$ in the current - voltage characteristics. Such $V_{R}$, which is associated with tunneling effects in RTD, could lead to tunable terahertz generation and detection for communication applications.
\end{abstract}

Keywords: nanowire, resonant tunneling diodes, quantum device simulator, physically terahertz applications

\section{INTRODUCTION}

An emerging area related to $5 \mathrm{G}$ technology development and application is terahertz communications [1,2]. The resonant tunneling diode (RTD) $[3,4,5]$ is a very promising device for wireless and optical terahertz generation and detection. The current planar RTD frequencies of operation and performance are determined by the layer design and growth of the RTD structures. However, there is a possibility to tune further the RTD behaviour by patterning and introducing gate control. Due to the simple design and the quantum phenomena associated with their operation, RTDs have been a very popular test-bed for the study of quantum mechanical effects in semiconductor devices. Theoretically, these devices have been a rather successful model for testing and benchmarking device simulation software and suites.

As for any exploratory study on electron devices and applications, computer simulation tools are the ones to pave the way for experimental device fabrication. The Device Modelling group at the University of Glasgow is actively developing a device simulation framework known as NanoElectronic Simulation Software (NESS) [6] [7], which simulates charge transport in ultra-scaled nanoelectronic devices with various integrated modules and solvers, such as

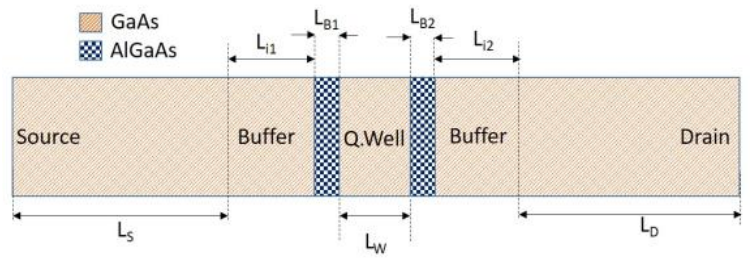

Figure 1. Schematic representation of the simulated RTD device where $\mathrm{L}_{\mathrm{s}}=\mathrm{L}_{\mathrm{d}}=19 \mathrm{~nm}, \mathrm{Li}_{1}=\mathrm{Li}_{2}=3 \mathrm{~nm}, \mathrm{~L}_{\mathrm{b}}=\mathrm{L}_{\mathrm{b}}=3 \mathrm{~nm}$ and $\mathrm{L}_{\mathrm{w}}=5 \mathrm{~nm}$. The barrier regions

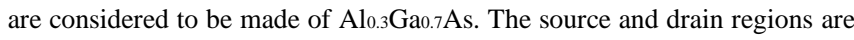
$\mathrm{n}$-type doped with concentration of $2 \times 1018 \mathrm{~cm}-3$, while in the rest of the device the doping is n-type with concentration of $1 \times 1015 \mathrm{~cm}-3$.

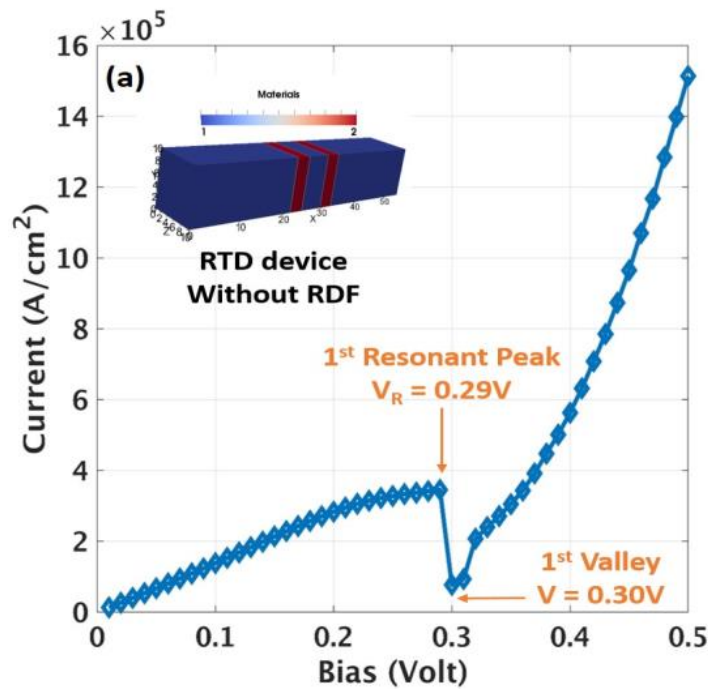

Figure 2. Current-voltage ( $\left.I_{D}-V_{D}\right)$ curve for the simulated 'smooth' device and the positions on the first resonant peak $-V_{R}$. 'Smooth' device is the device without any source of statistical variability, such as Random Dopants Fluctuation (RDF). The inner plot shows the device structure implemented

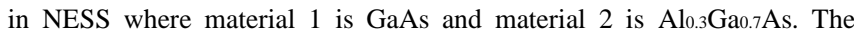
dimensions of the device in $\mathrm{nm}$ is also shown on the same plot. The $\mathrm{ID}_{\mathrm{D}} \mathrm{V}_{\mathrm{D}}$ curve shows the 1 st resonant peak at $0.29 \mathrm{~V}$ drain bias and after this there is the so called negative differential resistance region (NDR) where the current drops with increasing of the bias. The first valley is $0.30 \mathrm{~V}$ drain bias.

non-equilibrium Green's function (NEGF), drift-diffusion, and Kubo-Greenwood methods [7]. In this paper, we report a simulation study on tunable gated RTD device-based GaAs materials generated by creating two AlGaAs barriers in a nanowire structure. For the first time, we demonstrate that by applying gate voltage we can tune the RTD resonant peak position. 

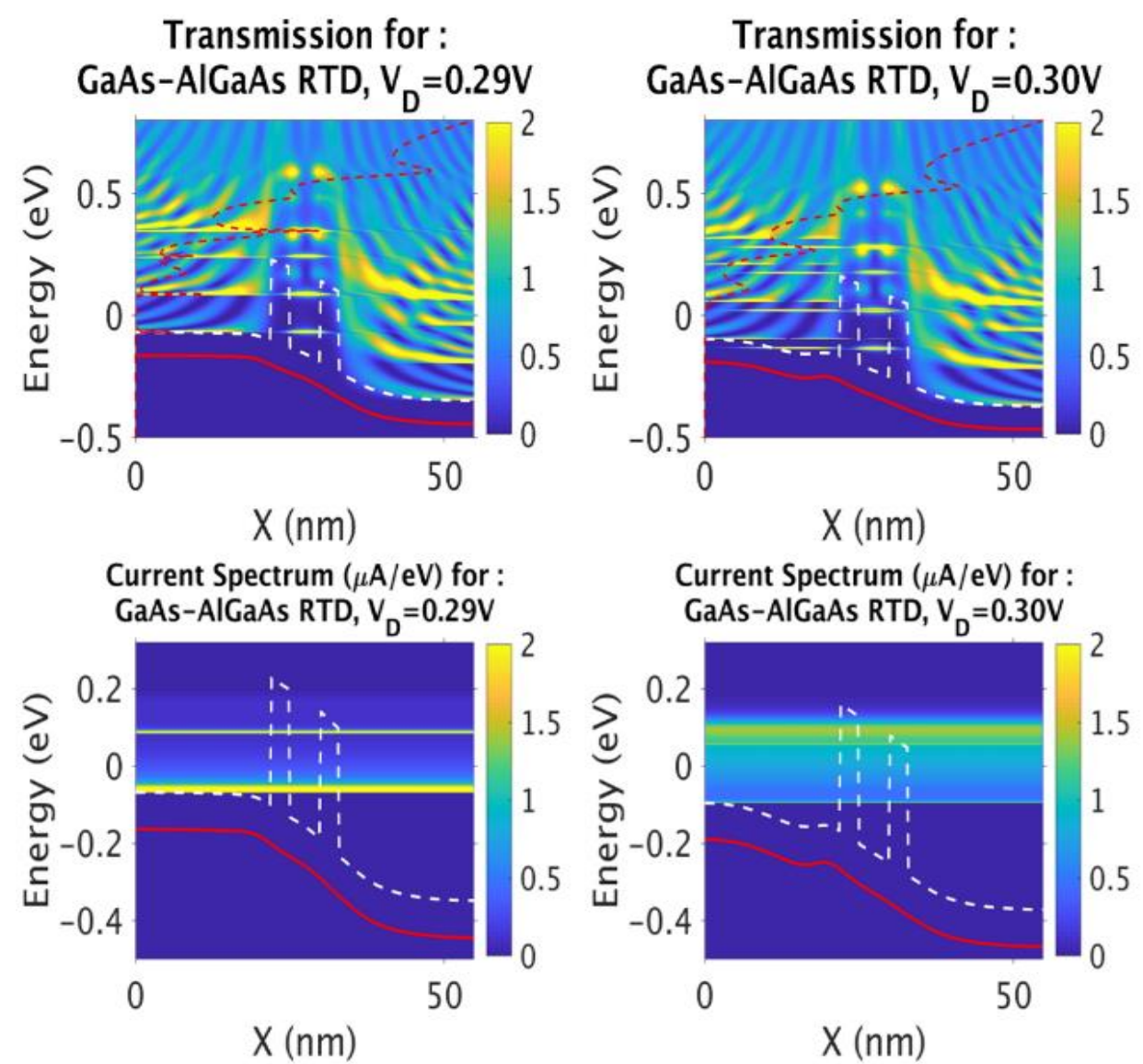

Figure 3. Top row: Local Density of States (LDOS), band diagram (white line), average potential (red solid line), transmission curves (red dashed lines (top). Bottom row: current spectra of the device at the two values of the drain voltage $\left(V_{D}\right): V_{D}=0.29 \mathrm{~V}$ and $0.30 \mathrm{~V}$.

\section{DEVICE DESCRIPTION AND SIMULATION METHODOLOGY}

The schematic view of the device considered herein is shown in Fig. 1. GaAs nanowire RTD has a square crosssection of $10 \mathrm{~nm}$ and a total length of $55 \mathrm{~nm}$. The dimensions with reference to Fig. 1(a) are $\mathrm{Ls}_{\mathrm{s}}=\mathrm{Ld}=19 \mathrm{~nm}, \mathrm{Li}_{1}=\mathrm{Li} 2=3 \mathrm{~nm}$, $\mathrm{Lb}_{1}=\mathrm{L} b 2=3 \mathrm{~nm}$, and $\mathrm{L}_{w}=5 \mathrm{~nm}$. The barrier regions are considered to be made of $\mathrm{Al} 0.3 \mathrm{Ga} 0.7 \mathrm{As}$. The source and drain regions are considered n-type doped with concentration of $2 \times 10_{18} \mathrm{~cm}-3$, whereas n-type concentration of $1 \times 1015 \mathrm{~cm}-3$ is taken in the rest of the device.

The simulation results presented herein are obtained using the NEGF solver implemented in the NESS framework [7]. For carrier transport, the recursive Green's function algorithm is employed considering effective mass approximation in the mode-space. The quantum transport is carried out selfconsistently with a numerical Poisson solver. The total carrier density determines the new potentials which are then fed back into the NEGF solver for a new current and charge distribution within the active region of the device. The self-consistent loop is repeated until the specific current convergence criterion is reached. For more details about the NESS software and capabilities please see [7].

\section{RESULTS AND DISCUSSION}

The output characteristic of the nanowire RTD provided in Fig. 2 shows the first resonant tunneling peak having a current value of $3.8 \times 105 \mathrm{~A} / \mathrm{cm}_{2}$ at a bias voltage of $0.29 \mathrm{~V}$, followed by a negative differential resistance region where the current drops to $7.7 \times 10_{4} \mathrm{~A} / \mathrm{cm}_{2}$ and thereafter increases. The results for the nanowire RTD device are qualitatively consistent with theoretical and experimental reports of bulk-devices made of similar materials, with similar thickness and doping profiles $[3,4,5]$. The LDOS plots shown in Fig. 3 for the resonance condition show the alignment of the quantum well energy levels with the source states at $-0.068,0.088$ and $0.346 \mathrm{eV}$ for the resonance voltage $\left(V_{R}\right)$ of $0.29 \mathrm{~V}$. When the LDOS of the source and quantum dot are aligned energetically, the transmission curves (the red dashed lines in Fig. 3) reveal sharp transmission peaks. This is an indication of tunneling of electrons trough the barriers from the source to the quantum dot.

This is in agreement with the current spectra, which is shown in the bottom part of Fig. 3, and it has two very well pronounced channels at the same energy where the transmission peaks are located. From Fig. 3, it is evident that the current is tunneling through the barrier. The LDOS alignment between the states from the source and the quantum dot is more disrupted as the bias changes to $0.30 \mathrm{~V}$. The current spectra in Fig. 3 also shows a more smeared nature at $0.30 \mathrm{~V}$ as compared to that at the resonance peak. Furthermore, the first two peaks of the transmission spectra are of much lower magnitude. This is also clearly visible in the $1 \mathrm{D}$ transmission plots in Fig. 3. This is due to the fact that the LDOS in the source and the quantum dot region are not aligned anymore. As a result, there is around one-order of magnitude decrease of the current from $0.29 \mathrm{~V}$ to $0.30 \mathrm{~V}$. 

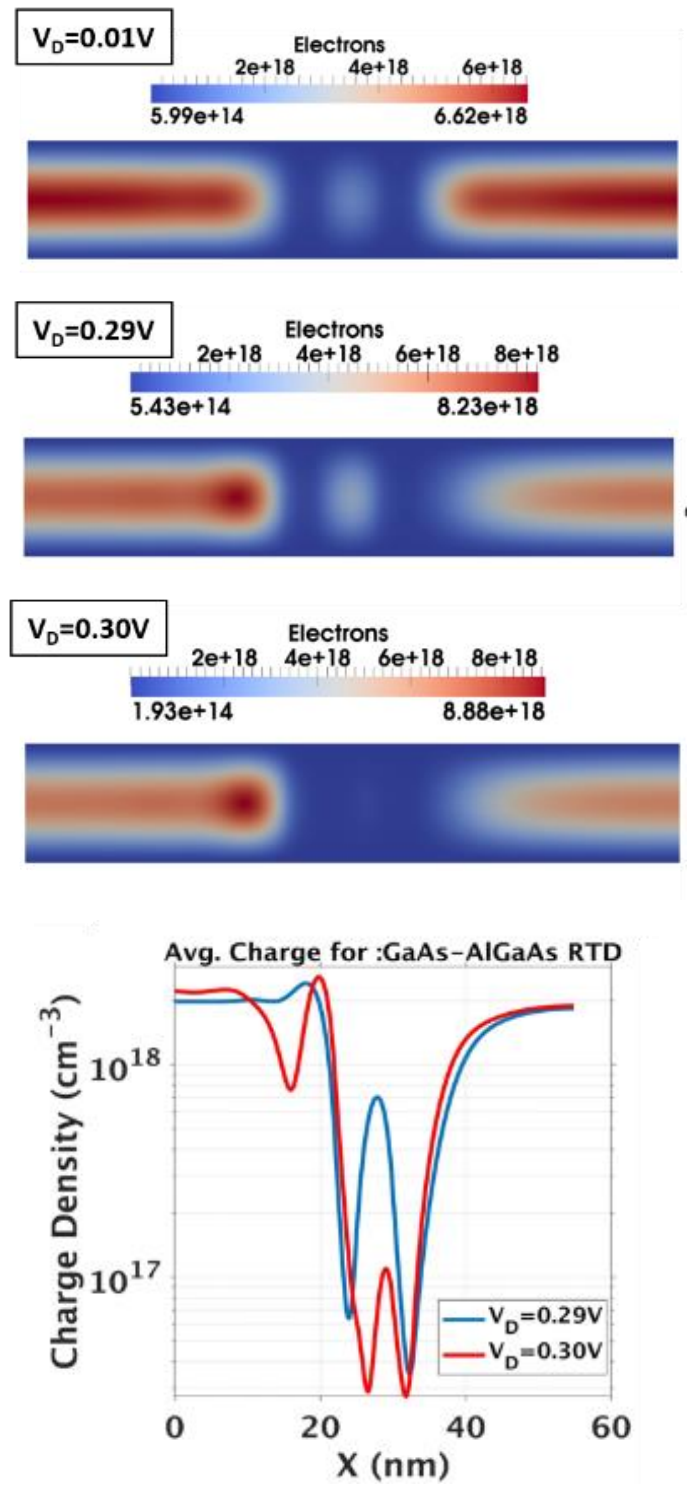

Figure 4. Top: charge density plots of the device at bias of $0.01 \mathrm{~V}$ (top), $0.29 \mathrm{~V}$ (middle), $0.30 \mathrm{~V}$ (bottom); Bottom: the average 1-D charge along the transport directions of the device for $\mathrm{V}_{\mathrm{D}}=0.29 \mathrm{~V}$ and $0.30 \mathrm{~V}$.

Top part of Fig. 4 shows the 2D plots of the charge distribution in the XY cut-plane in the middle of the device at different bias conditions. In particular, it shows how the charge distribution in the device changes as a function of the applied bias and the quantum well gets charged up to the resonance voltage of $0.29 \mathrm{~V}$ followed by a discharge at $0.30 \mathrm{~V}$. This is consistent with the data presented in Fig. 3 where it is clear that, at $0.29 \mathrm{~V}$, there are very pronounced transmission peaks at around $-0.1 \mathrm{eV}$ and $0.1 \mathrm{eV}$. At $0.30 \mathrm{~V}$ drain bias, due to change of the alignment between the LDOS in the contacts and the quantum well, the electrons cannot tunnel through the barrier and this leads to a significant drop in the current.

This is also manifested in the 1D profile of the charge density presented in Fig. 4. The charge inside of the well decreases with around one order of magnitude when the drain bias increases from $0.29 \mathrm{~V}$ to $0.3 \mathrm{~V}$ and, more interestingly, there is a significant charge drop just before the first barrier. The drop corresponds to the constructive interference coming from the

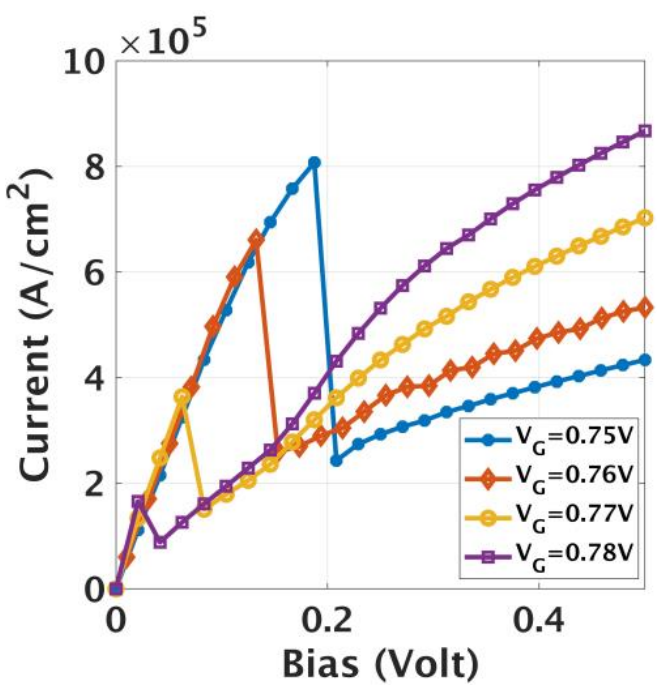

Figure 5. I-V characteristics of the proposed gated RTD with an ideal workfunction matched gate material showing the modulation of the resonance peak position with applied gate voltage.

wavefunctions that are reflected from the barrier because they cannot tunnel through. With the results simulated in NESS showing qualitative consistency in predicting output characteristics and capturing quantum phenomena in the RTD structure, we study the impact of the ideal work-function matched gate on the resonance voltage. For this purpose, a $2 \mathrm{~nm}$ thick $\mathrm{SiO}_{2}$ gate oxide was considered wrapped around the nanowire under an ideal surround gate of $17 \mathrm{~nm}$ in length (covering the buffer regions, the barriers and the quantum well).

From the simulation results presented in Fig. 5, it can be concluded that for a gate work function of $4.07 \mathrm{eV}$, the RTD behaviour may be only tuned on for voltages above $0.75 \mathrm{~V}$. Subject to a slight variation of $\mathrm{V}_{\mathrm{G}}$ in the $\mathrm{ON}$ condition, there can be a significant shift in $V_{R}$. As $V_{G}$ is changed from 0.75 to $0.78 \mathrm{~V}$, the value of $\mathrm{V}_{\mathrm{R}}$ is drastically shifted from $0.185 \mathrm{~V}$ to $0.02 \mathrm{~V}$. Such wide variation of the resonance peak with small changes in the gate voltage and the ability to control the RTD behaviour with gate bias advocates the potential use of such devices for tunable terahertz communication applications. The position of the resonant peak is determined by the alignment of the LDOS in the source and the quantum dot. The latter can be controlled by the gate voltage and therefore the RTD behaviour can be tuned.

Fig. 6 reveals simulations of 125 RTD devices, each of them with a unique distribution of random dopants fluctuation (RDF), introduced in the device. From this data, it is clear that there is a direct correlation between the number and the position of RDFs in the device, the position of the $V_{R}$, and the currentvoltage characteristics $\left(\mathrm{ID}_{\mathrm{D}}-\mathrm{V}_{\mathrm{G}}\right)$. The key figures of merit that can be extracted from the ID- $\mathrm{V}_{\mathrm{G}}$ simulations are presented in Fig. 7. Fig 7. (a) reveals the correlation between $V_{R}$ and the number of dopants. It can be concluded that devices with the same number of dopants have a huge variation of $V_{R}$. For example, devices with 10 dopants have variation of $V_{R}$ from $0.05 \mathrm{~V}$ all the way up to $0.61 \mathrm{~V}$. This can be explained by the specific position of each dopant in the $3 \mathrm{D}$ volume of the device. 

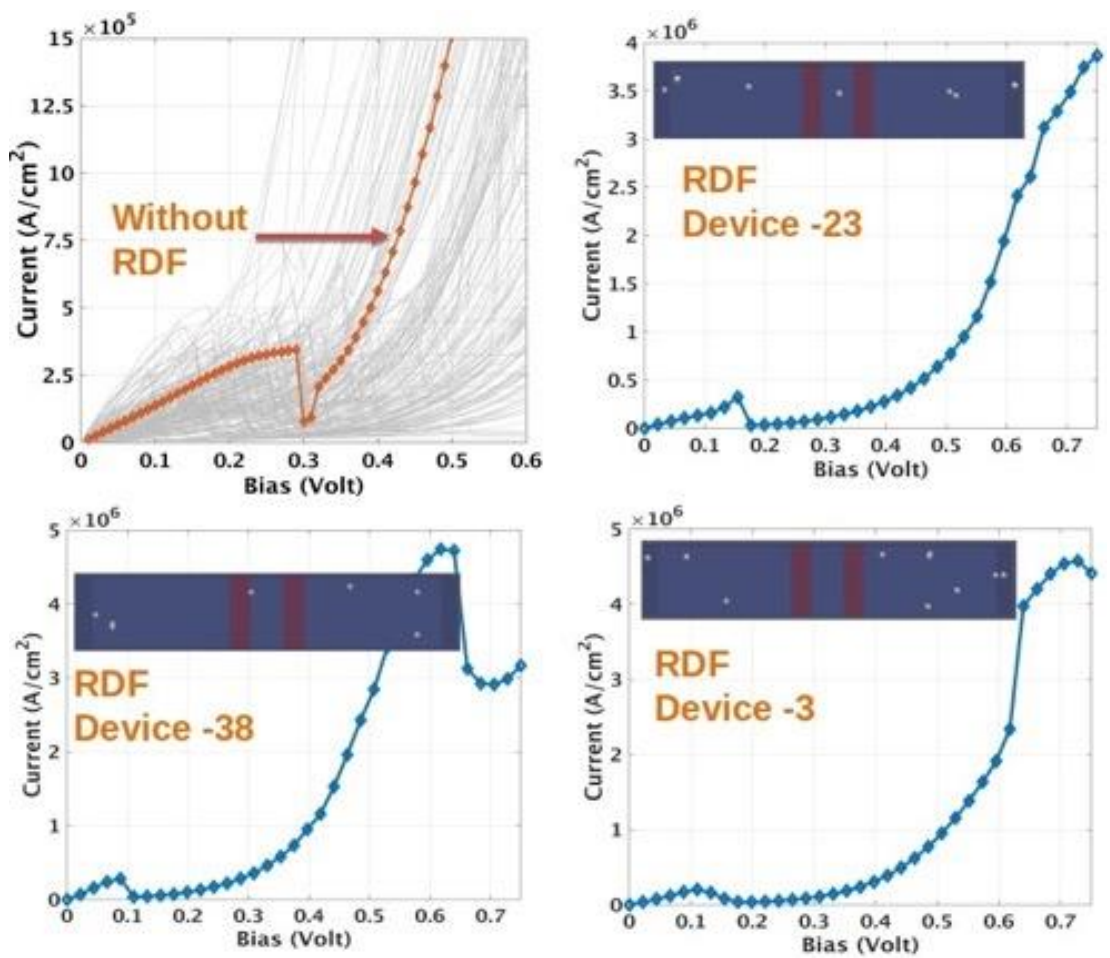

Figure 6. Current-voltage ( $\left.I_{D}-V_{D}\right)$ plots of the devices with the random dopant distribution (RDD). The device without any RDD (smooth device) is highlighted in orange for comparison purposes. ID-VD characteristics of RDF devices labelled as (b) device 23 (c) device 38 and (d) device 3 with inset device screenshots, where the exact location of the RDD in the structure can be seen as the grey spheres.
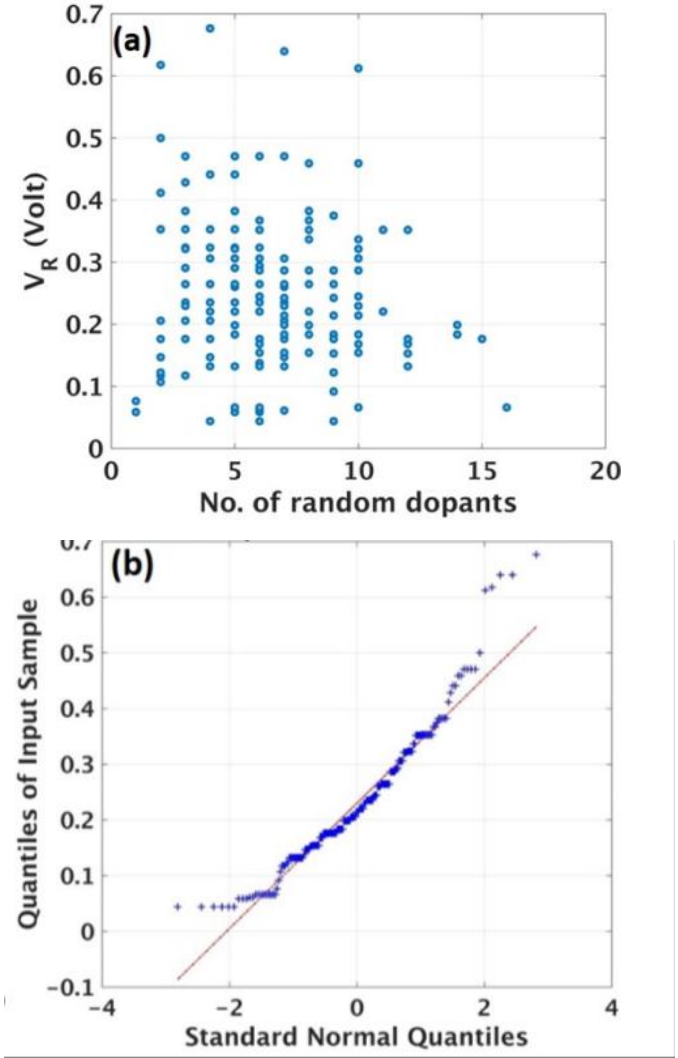

Figure 7. (a) Distribution of the first resonant peak voltage $\left(V_{R}\right)$ for the 125 devices as a function of the number of the random dopants. (b) Quantile-quantile (Q-Q) plot for the $V_{R}$ data.

Fig. 7. (b) is a QQ plot that shows the distribution of $V_{R}$ for all 125 RTD devices. It shows that there is a significant deviation from the normal distribution (red line) in the tail regions. This plot shows that it is not possible to use analytical models to describe the behaviour of automictically different RTDs.

\section{CONCLUSION}

Our work shows that quantum mechanical effects have a significant impact on the RTD devices. There is a direct link between the position of the resonance peak and the number and the position of the random dopants in the device. Indeed, these unique relationship opens a possibility for these devices to be used for tunable terahertz communication applications.

\section{REFERENCES}

[1] M. Feiginov, "Frequency Limitations of Resonant-Tunnelling Diodes in Sub-THz and THz Oscillators and Detectors," J. Infrared, Millimeter, Terahertz Waves, pp. 365-394, 2019.

[2] K. Kasagi, S. Suzuki, and M. Asada, "Large-scale array of resonanttunneling-diode terahertz oscillators for high output power at $1 \mathrm{THz}$, , J. Appl. Phys., vol. 125, no. 15, 2019.

[3] B. Biegel and J. Plummer, "Comparison of self-consistency iteration options for the Wigner function method of quantum device simulation," Phys. Rev. B - Condens. Matter Mater. Phys., vol. 54, no. 11, pp. 80708082, 1996.

[4] B. A. Biegel and J. D. Plummer, "Applied bias slewing in transient Wigner function simulation of resonant tunneling diodes," IEEE Trans. Electron Devices, vol. 44, no. 5, pp. 733-737, 1997.

[5] K. L. Jensen and F. A. Buot, "Numerical simulation of intrinsic bistability and high-frequency current oscillations in resonant tunneling structures," Phys. Rev. Lett., vol. 66, no. 8, pp. 1078-1081, 1991.

[6] S. Berrada, et al., "Quantum Transport Investigation of Threshold Voltage Variability in Sub-10 nm JunctionlessSi Nanowire FETs," 2018 International Conference on Simulation of Semiconductor Processes and Devices (SISPAD): Sept. 2018.

[7] S. Berrada et al., Nano-electronic Simulation Software (NESS): a flexible nano-device simulation platform. Journal of Computational Electronics, 19 , pp. $1031-1046,2020$ 